\title{
ISOMORPHISMS BETWEEN SEMIGROUPS OF ISOTONE MAPS
}

\author{
HANS-J. BANDELT \\ (Received 15 August 1979; revised 21 July 1980) \\ Communicated by T. E. Hall
}

\begin{abstract}
Every poset with 0 is determined by various semigroups of isotone selfmaps which preserve 0 . Two theorems along these lines are given and applied to some recent results concerning relation semigroups on topological spaces.
\end{abstract}

1980 Mathematics subject classification (Amer. Math. Soc.): primary 20 M 20; secondary 06 A 10 , $54 \mathrm{H} 15$.

\section{Introduction}

To any topological space there correspond various semigroups of maps or relations which are in a certain sense compatible with the topology. Of particular interest are those cases where an associated semigroup determines the space up to homeomorphism (see Gluskin and others (1977)). Schein (1970) has demonstrated that some results of this type are order theoretic in nature and thus follow from fairly general theorems on isomorphisms between semigroups of isotone maps. We will prove here (Theorem 2.2) a slight generalization of Schein (1970), Theorem 2, which also covers the main results of Magill (1969) and McAlister (1971).

\section{Two isomorphism theorems}

Let $P$ be a poset with 0 . By $\mathcal{E}_{P}$ denote the endomorphism semigroup of $(P,<, 0)$, that is the semigroup of all isotone maps $\varphi: P \rightarrow P$ such that $0 \varphi=0$. 
The range of a mapping $\varphi$ is denoted by $\operatorname{im} \varphi$; if $\operatorname{im} \varphi$ contains exactly two elements, then $\varphi$ is called 2-valued. We will say that a subsemigroup $S$ of $\mathscr{E}_{p}$ is sufficient in case it satisfies

(1) for every $x \in P$ there exists $\zeta \in S$ such that $\operatorname{im} \zeta=\{0, x\}$,

(2) if $x, y \in P$ and $x \$ y$, then there exists a 2-valued map $\zeta \in S$ such that $x \zeta \neq 0$ and $y \zeta=0$.

We note that every subsemigroup $S$ of $\varepsilon_{P}$ which is sufficient in the sense of Schein (1970) is also sufficient in our sense, but not conversely. Instead of (1), (2) Schein uses the conditions

(1') if $x, y \in P$ and $y \neq 0$, then there exists $\xi \in S$ such that $\operatorname{im} \xi=\{0, x\}$ and $y \xi=x$,

$\left(2^{\prime}\right)$ if $x, y \in P$ and $x \$ y$, then there exists $\varphi \in S$ such that $x \varphi \neq 0$ and $y \varphi=0$.

Clearly (1') implies (1). If $x \$ y$ in $P$, then by $\left(1^{\prime}\right),\left(2^{\prime}\right)$ there exist a map $\varphi \in S$ and a 2 -valued map $\xi \in S$ such that $x \varphi \xi=x \varphi \neq 0$ and $y \varphi=0$, whence the 2-valued $\operatorname{map} \zeta=\varphi \xi$ gives (2).

In what follows let $S$ be a sufficient subsemigroup of $\varepsilon_{P}$. For each $x \in P$ we can pick a map $\zeta_{x} \in S$ such that im $\zeta_{x}=\{0, x\}$. For $\varphi \in S$, the set $R(\varphi)=\{\psi$ $\in S \mid \varphi \cdot \psi=0\}$ is called the right annihilator of $\varphi$ in $S$.

2.1. LeMMA. Let $S$ be a sufficient subsemigroup of $\mathcal{E}_{P}$.

(i) $A$ map $\zeta \in S$ is the zero map or 2-valued if and only if for each $\varphi \in S$ either $\varphi \cdot \zeta=0$ or $R(\varphi \cdot \zeta)=R(\zeta)$.

(ii) For $x, y \in P, x \leqslant y$ if and only if for all 2-valued maps $\xi \in S, \zeta_{y} \cdot \xi=0$ implies $\zeta_{x} \cdot \xi=0$.

(iii) For $\varphi, \psi \in S, \varphi \leqslant \psi$ if and only if for all 2-valued maps $\zeta, \xi \in S$, $\zeta \cdot \psi \cdot \xi=0$ implies $\zeta \cdot \varphi \cdot \xi=0$.

Proof. Given any $\varphi, \psi \in S, R(\psi) \subseteq R(\varphi)$ holds if for each $x \in P$ there exists $y \in P$ with $x \varphi \leqslant y \psi$. On the other hand, if $\varphi, \zeta \in S$ such that $\operatorname{im} \zeta=\{0, y\}$ and there exists $x \in P$ with $x \varphi \$ y$, then by virtue of (2) we can find a 2-valued map $\xi \in S$ for which $\varphi \cdot \xi \neq 0$ and $\zeta \cdot \xi=0$; hence $R(\zeta) \underline{R} R(\varphi)$. Taking this into account, (ii) and (iii) are readily verified. Now to prove (i) let $\varphi, \zeta \in S$; if $\zeta$ is 2-valued, then clearly either $\varphi \cdot \zeta=0$ or $\varphi \cdot \zeta$ and $\zeta$ have equal images and hence equal right annihilators. Conversely, if $R\left(\zeta_{x} \cdot \zeta\right)=R(\zeta)$ for some $x \in P$, then the images of $\zeta_{x} \cdot \zeta$ and $\zeta$ must be equal, and so $\zeta$ is either the zero map or 2-valued.

2.2. Theorem. Let $P$ and $Q$ be posets with 0 and let $S$ and $T$ be sufficient subsemigroups of $\mathcal{E}_{P}$ and $\mathcal{E}_{Q}$ respectively. If $F: S \rightarrow T$ is an isomorphism, then 
there exists a unique isomorphism $\theta: P \rightarrow Q$ such that

$$
F(\varphi)=\theta^{-1} \cdot \varphi \cdot \theta \quad \text { for all } \varphi \in S .
$$

Proof. Trivially, $F(0)=0$ and by $2.1 F$ restricts to a bijection between the sets of 2-valued members of $S$ and $T$ respectively. If $\zeta \in S$ has the same image as $\zeta_{x}$, then $R(\zeta)=R\left(\zeta_{x}\right)$; consequently, the right annihilators of $F(\zeta)$ and $F\left(\zeta_{x}\right)$ in $T$ are equal, and so im $F(\zeta)=\operatorname{im} F\left(\zeta_{x}\right)$. Therefore the map $\theta: P \rightarrow Q$ defined by $\operatorname{im} F\left(\zeta_{x}\right)=\{0, x \theta\} \quad(x \in P)$ is a bijection and thus in view of 2.1 an isomorphism. For all $y \in Q$ and 2-valued maps $\zeta \in S$ we have im $F(\zeta)=$ $\operatorname{im} \theta^{-1} \cdot \zeta \cdot \theta$ and further

$$
y F(\zeta)=0 \Leftrightarrow F\left(\zeta_{y \theta^{-1}}\right) \cdot F(\zeta)=0 \Leftrightarrow \zeta_{y \theta^{-1}} \cdot \zeta=0 \Leftrightarrow y \theta^{-1} \cdot \zeta \cdot \theta=0 .
$$

Hence the equation (*) is true for all 2-valued maps $\varphi \in S$. Now given $\varphi \in S$ arbitrary, we infer for 2-valued maps $\zeta, \xi \in S$,

$\zeta \cdot \varphi \cdot \xi=\theta \cdot F(\zeta \cdot \varphi \cdot \xi) \cdot \theta^{-1}=\theta \cdot F(\zeta) \cdot F(\varphi) \cdot F(\xi) \cdot \theta^{-1}=\zeta \cdot \theta \cdot F(\varphi) \cdot \theta^{-1} \cdot \xi$. By 2.1 we therefore have $F(\varphi)=\theta^{-1} \cdot \varphi \cdot \theta$ as required. Computing $F\left(\zeta_{x}\right)$ for all $x \in P$, we see that $\theta$ is the unique isomorphism satisfying (*).

The preceding result generalizes Theorem 2 of Schein (1970). Comparing 2.2 with the results of McAlister (1971), we notice that by 2.1 a surjective homomorphism $F: S \rightarrow T$ between sufficient semigroups is an isomorphism if and only if for $\varphi \in S, F(\varphi)=0 \Leftrightarrow \varphi=0$.

Let us call a partial map $\varphi$ on a poset $P$ with domain $A$ isotone if $x \in A$, $y \in P, x \leqslant y$ implies $y \in A$ and $x \varphi \leqslant y \varphi$. A semigroup $S$ of isotone partial maps on $P$ is called sufficient if for every $x \in P$ there exists a constant partial map $\zeta_{x} \in S$ with value $x$ and if for every $x \notin y$ in $P$ there exists a constant partial map $\zeta \in S$ whose domain contains $x$ but not $y$.

Since for any poset $P$ with 0 there is an obvious 1-1 correspondence between the maps $\varphi \in \mathcal{E}_{P}$ and the isotone partial maps $\varphi$ on the poset $P-\{0\}$ (see Schein (1970), p. 46), 2.2 may be restated as

2.3. Corollary. Let $S$ and $T$ be sufficient semigroups of isotone partial maps on the posets $P$ and $Q$ respectively. Then every isomorphism $F: S \rightarrow T$ is induced by a unique isomorphism $\theta: P \rightarrow Q$.

An isotone map $\varphi$ on a poset $P$ is called residuated if there is another isotone map $\varphi^{+}$on $P$ such that $x \varphi^{+} \cdot \varphi \leqslant x \leqslant x \varphi \cdot \varphi^{+}$for all $x \in P$. The set $\operatorname{Res}(P)$ of all residuated maps on $P$ forms a semigroup (under composition). Moreover, if $P$ is bounded, then $\operatorname{Res}(P)$ is a sufficient subsemigroup of $\mathcal{E}_{P}$. This is due to the 
fact that all maps

$$
\zeta_{b}^{a}: x \mapsto \begin{cases}0 & \text { if } x \leqslant a, \\ b & \text { otherwise }\end{cases}
$$

with $a, b \in P$ belong to $\operatorname{Res}(P)$ (see Blyth and Janowitz (1972)). Hence from Theorem 2 of Schein (1970) or the above theorem one may deduce the following

2.4. Corollary. Let $P$ and $Q$ be bounded posets. Any isomorphism $F: \operatorname{Res}(P)$ $\rightarrow \operatorname{Res}(Q)$ is induced by a unique isomorphism $\theta: P \rightarrow Q$.

Thus, the semigroup $\operatorname{Res}(P)$ determines the bounded poset $P$ up to isomorphism-a fact which was also established by Johnson (1971).

Next we will prove a more general isomorphism theorem for semigroups of residuated mappings on complete lattices, which is not a consequence of 2.2. Henceforth let $L$ be a complete lattice. As is well known a map $\varphi$ on $L$ is residuated if and only if $\varphi$ preserves arbitrary joins (including 0 ). A subset $K$ of $L$ is called join dense if every element of $L$ is the join of some elements of $K$. We now call a subsemigroup $S$ of $\operatorname{Res}(L)$ sufficient if it contains the zero map and satisfies the following two conditions:

(3) the set $\{1 \zeta \mid \zeta \in S 2$-valued $\}$ is join dense in $L$,

(4) if $x, y \in L$ and $x \$ y$, then there exists a 2-valued map $\zeta \in S$ such that $x \zeta \neq 0$ and $y \zeta=0$.

The right annihilator of $A \subseteq S$ is the set $R(A)=\{\psi \in S \mid \varphi \cdot \psi=0$ for all $\varphi \in A\}$. Again we begin with a lemma.

2.5. Lemma. Let $S$ be a sufficient subsemigroup of $\operatorname{Res}(L)$. For each $x \in L$ put $A_{x}=\{\varphi \in S \mid 1 \varphi \leqslant x\}$.

(i) For $x, y \in L, x \leqslant y$ if and only if $R\left(A_{y}\right) \subseteq R\left(A_{x}\right)$.

(ii) $A$ map $\zeta \in S$ is 2-valued if and only if $\zeta \neq 0$ and for each $\varphi \in S$ either $\varphi \cdot \zeta=0$ or $R(\varphi \cdot \zeta)=R(\zeta)$.

(iii) For $\varphi, \psi \in S, \varphi \leqslant \psi$ if and only if for all 2-valued maps $\zeta, \xi \in S$, $\xi \cdot \psi \cdot \xi=0$ implies $\zeta \cdot \varphi \cdot \xi=0$.

(iv) $A$ subset $A$ of $S$ is equal to $A_{x}$ for some $x \in L$ if and only if $A$ is the left annihilator of $R(A)$.

Proof. If $x \leqslant y$, then $A_{x} \subseteq A_{y}$ and hence $R\left(A_{y}\right) \subseteq R\left(A_{x}\right)$. If $x \$ y$, then there exist $\varphi, \zeta \in S$ such that $1 \varphi \leqslant x, 1 \varphi \nless y, \varphi \cdot \zeta \neq 0, y \zeta=0$; hence $R\left(A_{y}\right) Z$ $R\left(A_{x}\right)$, proving (i). Since there are 2-valued maps $\zeta_{x} \in S$ with im $\zeta_{x}=\{0, x\}$ for elements $x$ from a join dense subset of $L$, we can verify (ii) and (iii) in essentially the same way as the corresponding assertions of 2.1. It remains to check (iv). If 
$A \subseteq S$, then $\psi \in R(A)$ if and only if $1 \varphi \cdot \psi=0$ for all $\varphi \in A$ or equivalently, $x \psi=0$ where $x$ is the join of all $1 \varphi(\varphi \in A)$. Given $\varphi \in S$ such that $1 \varphi \$ x$, (4) provides us with a map $\zeta \in S$ which satisfies $\varphi \cdot \zeta \neq 0$ and $x \zeta=0$; hence $\varphi$ is not in the left annihilator of $R(A)$. We conclude that $\{\varphi \in S \mid 1 \varphi \leqslant x\}$ is the left annihilator of $R(A)$, completing the proof.

2.6. Theorem. Let $L$ and $M$ be complete lattices and let $S$ and $T$ be sufficient subsemigroups of $\operatorname{Res}(L)$ and $\operatorname{Res}(M)$ respectively. Every isomorphism $F: S \rightarrow T$ is induced by a unique isomorphism $\theta: L \rightarrow M$.

Proof. By virtue of 2.5 we can define an isomorphism $\theta: L \rightarrow M$ by $F\left(A_{x}\right)=A_{x \theta}(x \in L)$ or equivalently, by $1 F(\varphi)=1 \varphi \cdot \theta$ for all $\varphi \in S$. Further, $F$ establishes a bijection between the 2-valued members of $S$ and $T$ respectively. If $\zeta \in S$ is 2-valued, then $1 F(\zeta)=1 \theta^{-1} \cdot \zeta \cdot \theta$ and for all $x \in L$,

$$
\begin{aligned}
x \theta \cdot F(\zeta) & =0 \Leftrightarrow \psi \cdot F(\zeta)=0 \text { for all } \psi \in A_{x \theta} \\
& \Leftrightarrow \varphi \cdot \zeta=0 \text { for all } \varphi \in A_{x} \\
& \Leftrightarrow x \zeta=0 \Leftrightarrow x \zeta \cdot \theta=0 .
\end{aligned}
$$

Hence $F(\zeta)=\theta^{-1} \cdot \zeta \cdot \theta$. An application of 2.5 settles the equation $F(\varphi)=\theta^{-1}$. $\varphi \cdot \theta$ for all $\varphi \in S$, and we are done.

For a complete lattice $L$, let $\Sigma(L)$ be the semigroup of all residuated maps $\varphi$ on $L$ such that $x \varphi \leqslant x$ for all $x \in L$. By virtue of Lemma 4 of Schreiner (1973), $\Sigma(L)$ is a sufficient subsemigroup of $\operatorname{Res}(L)$ if and only if $L$ is completely distributive. Hence 2.6 implies

2.7. Corollary. Let $L$ and $M$ be completely distributive complete lattices. Any isomorphism $F: \Sigma(L) \rightarrow \Sigma(M)$ is induced by a unique isomorphism $\theta: L \rightarrow M$.

\section{Sufficient semigroups of relations}

A binary relation $\rho$ on a set $X$ can be thought of as a mapping $\vec{\rho}$ on the power set of $X: A \vec{\rho}=A \rho=\{x \in X \mid(a, x) \in \rho$ for some $a \in A\}, A \subseteq X$. Thus to any relation semigroup there naturally corresponds a semigroup of isotone maps. Therefore the results of the preceding section immediately apply to semigroups of relations. $\mathscr{B}_{X}$ and $\mathscr{D}_{X}$ will denote the semigroups of all relations on $X$ and all dense relations on $X$ respectively; a relation $\rho \in \mathscr{G}_{X}$ is called dense whenever $X \rho=X \rho^{-1}=X($ Schein $(1970))$. 
Let $S$ be a subsemigroup of $\mathscr{B}_{X}$ such that $\varnothing \in S$. Put

$$
\begin{gathered}
\widetilde{\mathscr{F}}_{S}=\{A \subseteq X \mid A \times B \in S \text { for some } \varnothing \neq B \subseteq X\}, \\
\mathcal{G}_{S}=\{B \subseteq X \mid A \times B \in S \text { for some } \varnothing \neq A \subseteq X\} .
\end{gathered}
$$

If $S$ satisfies the conditions

(5) for all $x \in X,\{x\} \in \mathcal{G}_{S}$,

(6) for every $B \in \mathcal{G}_{S}$ and $x \notin B$ there exists $A \in \mathscr{F}_{S}$ such that $x \in A$ and $A \cap B=\varnothing$,

then $S$ is called a sufficient subsemigroup of $\mathscr{B}_{X}$.

A semigroup of dense relations is never sufficient in the preceding sense. Instead, we call a subsemigroup $S$ of $\mathscr{D}_{X}$ sufficient if $X \times X \in S$ and $S$ satisfies (5) and (6) for the associated sets

$$
\begin{gathered}
\mathscr{F}_{S}^{\prime}=\{A \subseteq X \mid A \times X \cup X \times B \in S \text { for some } B \subseteq X\}, \\
\mathcal{G}_{S}^{\prime}=\{B \subseteq X \mid A \times X \cup X \times B \in S \text { for some } A \subseteq X\} .
\end{gathered}
$$

If $S$ is a sufficient subsemigroup of $\mathscr{B}_{X}$ or $\mathscr{D}_{X}$, then the semigroup $\vec{S}$ of all $\vec{\rho}$ : $\mathcal{G}_{S} \rightarrow \mathcal{G}_{S}$ or $\vec{\rho}: \mathcal{G}_{S} \rightarrow \mathcal{G}_{S}^{\prime}(\rho \in S)$ is a sufficient subsemigroup of $\mathcal{E}_{P}$, where $P$ denotes the poset $\left(\mathcal{G}_{S}, \subseteq, \varnothing\right)$ or $\left(\mathcal{G}_{S}^{\prime}, \supseteq, X \times X\right)$, respectively. Now, from 2.2 one obtains without difficulty

3.1. Theorem. Let $S$ and $T$ be sufficient subsemigroups of $\mathscr{B}_{X}$ and $\mathscr{G}_{Y}\left(\right.$ or, $\mathscr{O}_{X}$ and $\mathscr{D}_{Y}$ ), then for every isomorphism $F: S \rightarrow T$ there exists a unique bijection $\alpha$ : $X \rightarrow Y$ such that $F(\rho)=\alpha^{-1} \cdot \rho \cdot \alpha$ for all $\rho \in S$.

Proof. By 2.2, $F$ is induced by an isomorphism $\varphi: \mathcal{G}_{S} \rightarrow \mathcal{G}_{T}\left(\varphi: \mathcal{G}_{S}^{\prime} \rightarrow \mathcal{G}_{T}^{\prime}\right.$, respectively). Then the bijection $\alpha: X \rightarrow Y$ defined by $\{x \alpha\}=\{x\} \varphi(x \in X)$ induces $\varphi$, whence $F(\rho)=\alpha^{-1} \cdot \rho \cdot \alpha$ for all $\rho \in S$.

A subsemigroup $S$ of $\mathscr{B}_{X}$ which contains all relations $\{(x, x)\}(x \in X)$ is called a triform semigroup by Magill (1969) or an $r$-semigroup on $X$ by McAlister (1971). Hence 3.1 generalizes Theorem 2.2 of Magill (1969); and since a surjective homomorphism $F: S \rightarrow T$ between sufficient subsemigroups of $\mathfrak{B}_{X}$ and $\mathscr{B}_{Y}$ is an isomorphism in case $F(\rho)=0 \Leftrightarrow \rho=0(\rho \in S), 3.1$ also establishes McAlister (1971), Theorem 6.

The typical application of 3.1 is the production of various isomorphism theorems for relation semigroups on topological spaces. To illustrate this point we will have a brief look at semigroups of continuous relations. A relation $\rho$ on a topological space $X$ is called continuous if $A \rho^{-1}$ is open whenever $A$ is an open set in $X, A \rho^{-1}$ is closed whenever $A$ is a closed set in $X$, and if $\{x\} \rho$ is compact for all $x \in X$. The set $C(X)$ of all continuous relations on $X$ is a subsemigroup of $\mathscr{B}_{X}$ (see Bednarek and Norris (1977)). Evidently, $\mathscr{F}_{C(X)}$ is the set of all clopen 
sets and $\mathcal{G}_{C(X)}$ is the set of all compact sets in $X$. Thus, whenever $X$ is a 0-dimensional Hausdorff space, $C(X)$ is a sufficient subsemigroup of $\mathscr{B}_{X}$. Applying 3.1, we can therefore improve Theorem 4.7 of Bednarek and Norris (1977):

3.2 Corollary. Let $X$ and $Y$ be 0-dimensional Hausdorff spaces. Then every isomorphism $F: C(X) \rightarrow C(Y)$ is induced by a unique homeomorphism $\alpha: X \rightarrow Y$.

Proof. The bijection $\alpha$ given by 3.1 induces a bijection between the sets of clopen sets in $X$ and $Y$ respectively, and thus is a homeomorphism.

The analogous isomorphism theorem, of course, holds for the semigroups of all continuous dense relations. All this may also be derived from Theorem 2 of Schein (1970). His Theorem 1 settles another case which is not attainable by our results. Call a relation $\rho \in \mathscr{B}_{X}$ wide if $X \rho^{-1}=X$; for a topological space $X$ let $\mathrm{CW}(X)$ denote the semigroup of all continuous wide relations on $X$. By $\mathcal{K}(X)$ denote the set of all non-empty compact sets in $X . \mathrm{CW}(X)$ corresponds to the semigroup $\mathrm{CW}(X)$ of all $\vec{\rho}: \mathscr{K}(X) \rightarrow \mathscr{K}(X), \rho \in \mathrm{CW}(X)$. Since for every clopen set $A$ and compact sets $B_{1} \subseteq B_{2}$ in $X$, the relation $A \times B_{1} \cup(X-A) \times B_{2}$ is continuous and wide, for any 0-dimensional Hausdorff space $X, \overrightarrow{C W}(X)$ is a sufficient subsemigroup of the semigroup of all isotone maps on $\mathscr{K}(X)$ in the sense of Schein (1970). Hence we arrive at the following result which partially answers a question of Bednarek and Norris (1977).

3.3. Proposition. Let $X$ and $Y$ be 0-dimensional Hausdorff spaces. Then every isomorphism $F: \mathrm{CW}(X) \rightarrow \mathrm{CW}(Y)$ is induced by a unique homeomorphism $\alpha$ : $X \rightarrow Y$.

Proof, From Schein (1970), Theorem 1 we infer that $F$ must either be induced by an isomorphism or a dual isomorphism between $\mathscr{K}(X)$ and $\mathscr{K}(Y)$. Since the latter case is obviously impossible, we finally get the desired homeomorphism $\alpha$ as in 3.2 .

The requirement for a subsemigroup $S$ of $\mathscr{B}_{X}$ that all singletons be in $\mathscr{G}_{S}$ (or $\mathscr{F}_{S}$ ) is not always necessary to prove a theorem analogous to 3.1. For, consider a locally compact 0 -dimensional space $X$. The lattice $\mathfrak{E}_{X}$ of all compact open sets in $X$ is a generalized Boolean lattice whose Stone space is homeomorphic to $X$ (see Grätzer (1978)). Because of the Stone duality any isomorphism between such lattices $\mathfrak{L}_{X}$ and $\mathfrak{L}_{Y}$ is induced by a unique homeomorphism $\alpha: X \rightarrow Y$. 
Hence 2.2 implies

3.4. Corollary. Let $X$ and $Y$ be locally compact 0-dimensional spaces. Then every isomorphism between the semigroups of all compact open relations on $X$ and $Y$ is induced by a unique homeomorphism of $X$ onto $Y$.

For compact 0-dimensional spaces 3.4 specializes to the isomorphism theorem for the semigroups of all clopen relations on Boolean spaces, which was proved by Bednarek and Magill (1976).

\section{References}

A. R. Bednarek and K. D. Magill Jr. (1976), 'Semigroups of clopen relations', Rev. Roum. Math. Pures Appl. 21, 1183-1188.

A. R. Bednarek and E. M. Norris (1977), 'Two semigroups of continuous relations', J. Austral. Math. Soc. Ser. A 23, 46-58.

T. S. Blyth and M. F. Janowitz (1972), Residuation theory (Pergamon Press).

L. M. Gluskin, B. M. Schein, L. B. Sneperman and I. S. Yaroker (1977), 'Addendum to a survey of continuous selfmaps', Semigroup Forum 14, 95-125.

G. Grätzer (1978), General lattice theory (Akademie-Verlag).

C. S. Johnson Jr. (1971), 'Semigroups coordinatizing posets and semilattices', J. London Math. Soc. 4, 277-283.

K. D. Magill Jr. (1969), 'Isomorphisms of triform semigroups', J. Austral. Math. Soc. 10, 185-193.

D. B. McAlister (1971), 'Homomorphisms of semigroups of binary relations', Semigroup Forum 3, 185-188.

B. M. Schein (1970), 'Ordered sets, semilattices, distributive lattices and Boolean algebras with homomorphic endomorphism semigroups', Fund. Math. 68, 31-50.

E. A. Schreiner (1973), 'Tight residuated mappings', Proc. Univ. of Houston, Lattice Theory Conf., pp. 519-530.

FB IV-Mathematik

Universität Oldenburg

D-2900 Oldenburg

West Germany 